\title{
DESARROLLO DE UN OBJETO VIRTUAL DE APRENDIZAJE (OVA) 3D QUE PERMITA CONOCER Y APRENDER SOBRE LOS SERVICIOS QUE BRINDA LA UPCA
}

\author{
DEVELOPMENT OF A VIRTUAL LEARNING OBJECT (OVA) 3D THAT \\ ALLOWS TO KNOW AND LEARN ABOUT THE SERVICES PROVIDED BY \\ THE UPCA
}

\author{
MS. Francisco Chinchilla Torres, Ing. Yilber Jose Toro Manosalva **
}

\footnotetext{
* Universidad Popular del Cesar, seccional Aguachica, docente catedrático, semillero de investigación MICROSYSTEMS Grupo GIDEATIC. Ingeniero electrónico, especialista en docencia universitaria, magister en control, de procesos industriales.

E-mail: franciscochinchilla@unicesar.edu.co.
**Universidad Popular del Cesar, seccional Aguachica, semillero de investigación MICROSYSTEMS Grupo GIDEATIC. Ingeniero de sistemas. Aguachica, cesar, Colombia.
E-mail: yilberjose5@gmail.com

\begin{abstract}
Resumen: El presente proyecto tuvo como objetivo el desarrollo de un apoyo didáctico que facilitara la enseñanza al proceso de inducción y la asignatura de cátedra upecista. Se realizó el desarrollo de un (OVA) plasmado en un videojuego 3D, el cual dio a conocer las funciones y beneficios que las oficinas le brindaban a cada uno de los estudiantes de la UPCA. Para este proyecto se implementó la metodología ADDIE, mediante ella se realizó una encuesta de satisfacción con una muestra poblacional de 144 estudiantes de 230. Una vez realizados los cálculos del tamaño de la muestra, el $94 \%$ de la población equivalente a 135 estudiantes del primer semestre, pertenecientes a los diferentes programas, el OVA fue objeto de satisfacción, teniendo un nivel de confianza del 95\%. La implementación del OVA sirvió de herramienta lúdica virtual a estudiantes de la asignatura pertinente, y como una ayuda didáctica al docente.
\end{abstract}

Palabras clave: ADDIE, Didáctica, Lúdica, UPCA, Videojuego.

\begin{abstract}
The objective of this degree project was to develop a didactic support that facilitates the teaching of the induction process and the subject of the upecist chair. The development of an OVA was carried out in a 3D videogame, which revealed the functions and benefits that the offices offered to each of the students of the UPCA. For this project the ADDIE methodology was implemented, through which a satisfaction survey was conducted with a population sample of 144 students of 230. Once the sample size calculations were made, $94 \%$ of the population equivalent to 135 students of the first semester, belonging to the different programs, the OVA was object of satisfaction, having a confidence level of $95 \%$. The implementation of the OVA served as a virtual leisure tool for students of the relevant subject, and as a teaching aid to the teacher.
\end{abstract}

Keywords: ADDIE, Didactic, Playful, UPCA, Videogame 


\section{INTRODUCCIÓN}

Hoy en día los objetos virtuales de aprendizaje han venido poco a poco revolucionando la manera clásica de la enseñanza en las aulas de clase, estos cuentan con un gran respaldo por parte de la investigación e innovación en pedagogía, logrando así la inclusión de las TICS en el aprendizaje. Para nosotros lo anteriormente descrito es una de nuestras más grandes motivaciones para este proyecto ya que las nuevas herramientas que existen pueden mejorar la calidad de aprendizaje de las nuevas generaciones, logrando así instaurar nuevos modelos educativos que de una $\mathrm{u}$ otra manera fortalezca el aprendizaje significativo de los estudiantes (L Márquez, YA Lara, F Ángulo, 2017), (LOT Tellez, MPS Delgado, 2017). Es por esto, que el presente proyecto, es de gran importancia en la medida que con la creación de un Objeto Virtual de Aprendizaje (OVA) los estudiantes de primer semestre, como también los profesores de inducciones y cátedra upecista, pueden apropiarse de las diferentes herramientas que les brinda las nuevas tecnologías. Para tal fin, el proyecto fue realizado en varias fases los cuales nos permitieron construir un videojuego educativo, lo que dio como resultado la unión de las nuevas tecnologías con una dinámica pedagógica y didáctica, de lo que significa la formación en la educación superior sustentada en el uso de tecnologías de la información y comunicación.

\section{MATERIALES Y MÉTODOS}

La investigación se llevó a cabo en la universidad popular del cesar, sede Aguachica ubicada en el municipio de Aguachica departamento del cesar con dirección carrera $40 \mathrm{~km} 1$ vía al mar, de igual manera la recopilación de fotografías para el modelado virtual necesario se realizó en las instalaciones de la universidad. Esta investigación fue de tipo aplicada con enfoque cualitativo ya que buscaba generar conocimiento con aplicación directa tanto para identificar problemas sobre los que se debe intervenir, como para definir las estrategias de solución, al igual que la recopilación de información que se utilizara para el desarrollo de la ova multimedia, en este proyecto se implementó el modelo METODOLOGICO ADDIE el cual nos guio en las diferentes fases pertinentes para el desarrollo del video juego. Este modelo propone como alternativa la organización de las actividades que guíe el aprendizaje autónomo del estudiante, tal como se describe en las cinco fases que componen al modelo:

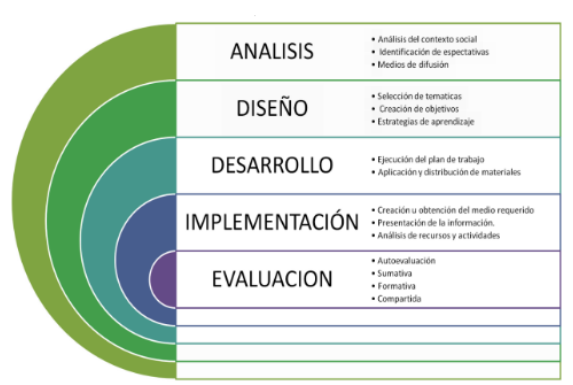

Fuente: [Base de datos en línea]: http://eldisenoinstruccionaleteg502.blogspot.com.co/2013/12/modelos-deldiseno-instruccional.html fecha 29 marzo, hora 9:32 am, 2018.

\section{ESQUEMA TEMATICO CAPITULO I.}

\section{IDENTIFICACIÓN DE LOS CONTENIDOS Y COMPONENTES ADECUADOS PARA EL DESARROLLO DEL OVA CON CONTENIDO MULTIMEDIA.}

Para el desarrollar y crear la ova se requeria utilizar una serie de programas que permitiera ejecutar y llevar acabo el desarrollo en su totalidad estos fueron: Blender, Unity 3d, Adobe After Effects, Adobe Fuse Cc (Beta), Anime Maker.

Para crear el video juego, se requiere utilizar de materiales tecnológicos e informáticos y material humano, los cuales se muestran a continuación:

\section{Tabla 1. RECURSO HUMANO}

\begin{tabular}{|c|c|}
\hline \multicolumn{2}{|r|}{ Recursos Humanos } \\
\hline $\begin{array}{l}\text { Yilber Jose Toro } \\
\text { Manosalva }\end{array}$ & $\begin{array}{l}\text { Estudiante de ingeniería de sistemas, Diseñador e } \\
\text { integrador de multimedia. }\end{array}$ \\
\hline $\begin{array}{l}\text { Leidy Johanna } \\
\text { Mejia Chogo }\end{array}$ & Estudiante de ingeniería de sistemas, Diseñadora Gráfica. \\
\hline $\begin{array}{l}\text { William } \\
\text { Contreras }\end{array}$ & $\begin{array}{l}\text { Artista de efectos visuales, laboro en la producción de } \\
\text { películas como, Valkiria: El amanecer del cuarto Reich, }\end{array}$ \\
\hline Huelsemann & $\begin{array}{l}\text { Biología 2.0: evolución evolutiva, Friend Request, } \\
\text { Northmen: Los Vikingos, Tarzán, entre otras, apoyara el } \\
\text { proyecto en la parte logística. }\end{array}$ \\
\hline $\begin{array}{l}\text { Mauricio Mejía } \\
\text { Chogó. }\end{array}$ & $\begin{array}{l}\text { Tecnólogo en mantenimiento de equipos, diseño e } \\
\text { instalación de cableado estructurado. Ing. Agroindustrial. } \\
\text { Asesor externo del proyecto "metodólogo". }\end{array}$ \\
\hline $\begin{array}{l}\text { Francisco } \\
\text { Chinchilla }\end{array}$ & Director técnico \\
\hline
\end{tabular}

Fuente: de los autores. 
Tabla 2. RECURSOS MATERIALES E INFORMÁTICOS

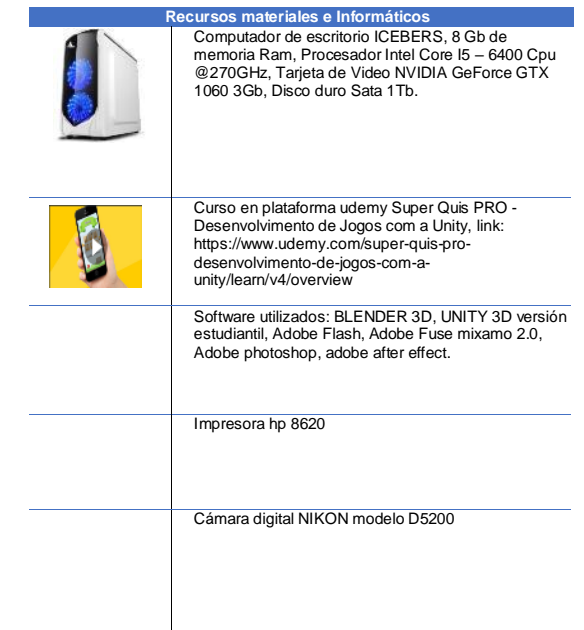

Fuente: recursos propios de los autores. Software en línea, licencia estudiantil gratis de la universidad. Y compra de licencia udemy super quis pro.

Durante el proceso de desarrollo y creación del video juego se llevó acabo, la recolección de información en las diferentes dependencias que tiene la universidad popular del cesar, tales como: bienestar institucional, registro y control, financiera, biblioteca, coordinación académica, egresados, internacionalización, coordinación de investigación y proyectos, a estos se les realizo una entrevista donde se preguntaban sobre las funciones que cumplen y que dudas eran más frecuentes por parte de los estudiantes.

\section{RESULTADOS Y GRÁFICOS.}

\section{CAPITULO II CONSTRUCCIÓN DEL DISEÑO EN TRES DIMENSIONES DEL CAMPUS DE LA UNIVERSIDAD POPULAR DEL CESAR SECCIONAL AGUACHICA.}

\section{Recopilación fotográfica y estructural del campus universitario.}

Se llevó acabo la recopilación fotográfica del campus universitario con una cámara fotográfica Nikon D5200, con la cual se tomaron imágenes en diferentes ángulos de toda la universidad, para tener una base inicial de la infraestructura y llevar acabo la creación del diseño en tres dimensiones de esta.

\section{Modelación y digitalización del campus universitario en Blender3D.}

Esta fase del proyecto se llevó a cabo luego de tener claro toda la información, fotos y videos necesarios en cuanto a la infraestructura de la universidad, tales como son fotografías de los salones, pupitres, plazoleta entre otras para luego proceder a su digitalización.

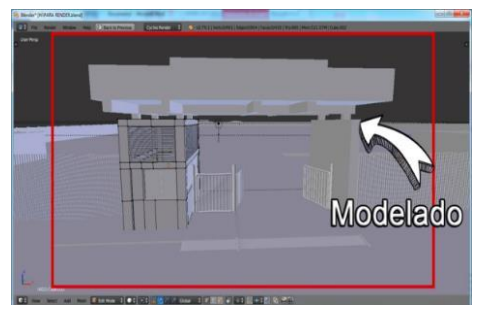

Fig. 1. Modelado y digitalización en blender3d.

Texturizado del modelado del campus universitario.

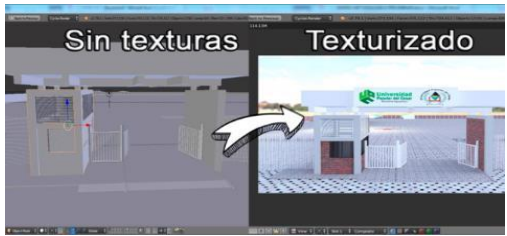

Fig. 2. Texturizado del modelado en $3 d$.

En este proceso se llevó a cabo la asignación de texturas, colores y de más materiales necesarios para lograr el hiperrealismo que se implementara en los videos de enseñanza del ova con contenido multimedia (video juego).

\section{CAPITULO III. CREACIÓN DE LAS ANIMACIONES GRAFICAS ADECUADAS A LOS MODELOS 3D PREESTABLECIDOS.}

\section{Animación de cámara de recorrido virtual}

En esta fase se realizaron las animaciones de recorrido de cámara virtual en el software Blender3D con el motor de renderizado CYCLES, con esto se buscó realizar un video hiperrealista que buscara semejar lo más posible el desplazamiento real que tiene que hacer un estudiante poder dirigirse a cualquiera de la dependencia deseada. 


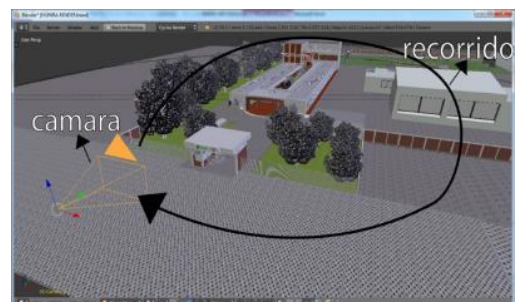

Fig. 3. Animación cámara de recorrido virtual en blender3d.

(Formatos, Reglamentos, Funciones, Deberes, Derechos, Etc.)

Después de haber recolectado la información de cada una de las entrevistas que se les hizo a los encargados de las dependencias académicas que dispone la universidad. Estas animaciones se realizaron en la aplicación web en la nube ANIMAKER el cual es utilizado para animaciones 2D profesionales. Dentro de la plataforma Animaker se crearon una serie de plantillas que nos permitieron diseñar cada uno de los videos.

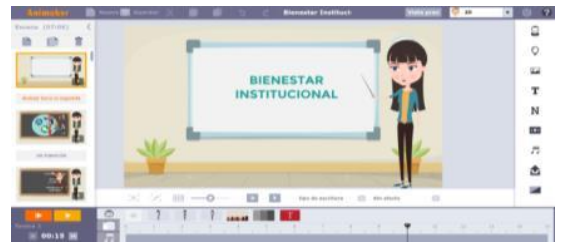

Fig. 4. Creación de videos en animaker.

Implementación de modelos 3d.

Una vez terminado el modelado, texturizado y aplicación de materiales al campus universitario en Blender3D, se realizó la exportación de todo el modelo a formato. FBX nativo de Unity3D.

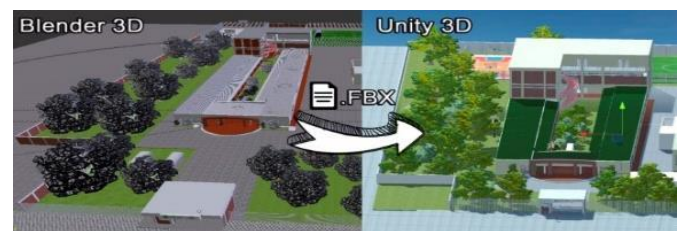

Fig. 5. Exportación De Modelo Del Campus Universitario De Blender3d A Unity3d

Importación De Las Animaciones Realizadas En Animaker 2d.

Una vez terminado las animaciones tanto de Blender3D en el motor de renderizado CYCLES, como también en Animaker 2D, se procedió a importarlas al proyecto de Unity 3D, para así utilizarlas en cada de las fases propuestas en el funcionamiento del OVA.

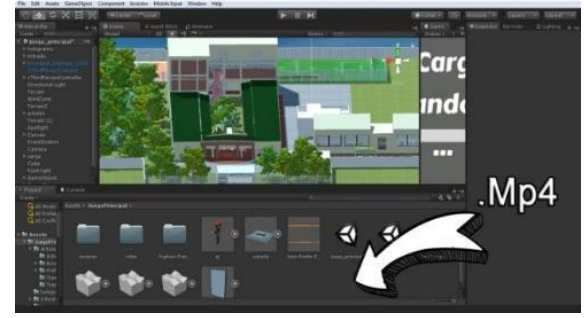

Fig. 6. Importación en formato $\mathrm{mp} 4$ videos educativos en animaker $2 d$.

\section{Mini Juego creado en Unity2D.}

En esta fase se desarrolló un Mini juego con el fin de evaluar el conocimiento que los estudiantes adquirieron al finalizar la enseñanza de los videos educativos, para esto se implementó la nueva herramienta de Unity que se viene utilizando de las últimas versiones de este software en cual es denominado CANVAS implementando códigos de programación en lenguaje c\#. Cada dependencia manejaba su propio mini juego con preguntas enfocadas exclusivamente a la zona académica administrativa que estuviese en proceso de aprendizaje. Este Mini juego constaba de diferentes funciones y propiedades que tornaban la ova más interesante y entretenido, las zonas académicas administrativas que contaban con 4 opciones de respuestas a una pregunta como también respuestas de falso y verdadero se implementó la función preguntas aleatorias, con el fin de que el mini juego tomara de 20 preguntas posibles 10 o 5 de ellas según la necesidad de acuerdo a lo extenso que estuviese la información de la zona, por otro lado también se implementó la función de tiempo límite para responder, a cada pregunta se le asignó un tiempo máximo de 40 segundos.

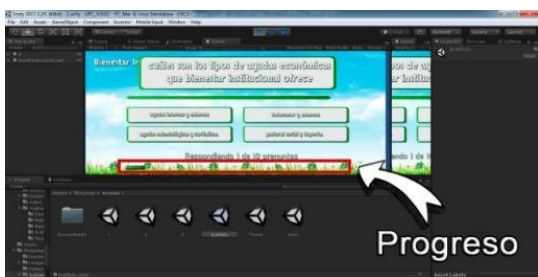

Fig. 7. Barra de tiempo preguntas minijuego unity $2 d$

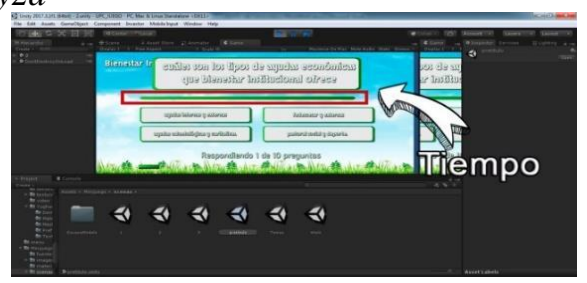

Fig 8. Barra De Progreso Preguntas Minijuego Unity $2 d$ 
En cuanto a la calificación final que se debía obtener por parte del usuario para poder superar la dependencia que estuviese jugando era de 3 estrellas, lo cual con 8 preguntas correctas se lograban obtener, si por lo contrario el estudiante obtenía menos de las 3 estrellas requeridas el estudiante debía que volver a repetir las preguntas hasta conseguir la calificación solicitada. Una vez obtenidas la calificación requerida para superar el nivel, esta dependencia automáticamente era marcada como superada y desaparecía de las opciones en el juego principal, dando paso a que el estudiante pudiese explorar en zonas diferente (MC Bonfante, A Castillo, 2017).

Creación de personaje Fuse y animación de personaje con Fuse CC y Máximo 2.0.

En esta fase del proyecto se realiza la creación del personaje que se utilizaría en el OVA, este fue realizado en el software para creación de personajes 3D Adobe fuse C.C, en este software se crea el personaje desde 0 , creando primero la cabeza, seguido del torso, luego los brazos y piernas, por último, se le aplicaron las vestimentas que más se nos adecuaron al rol de estudiante.

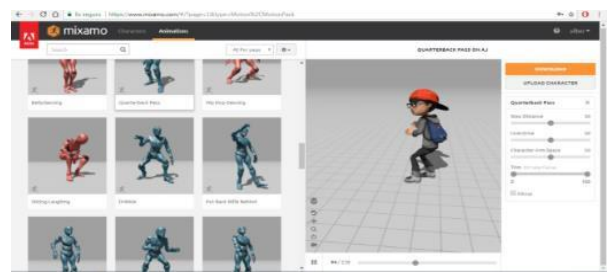

Fig. 9. Animación de personaje en máximo 2.0

Al terminar de haber aplicado las animaciones correspondientes a nuestro personaje se procedió a descargar cada una de las animaciones para así programarla con las funciones de Mecanim, herramienta interna del software Unity3D que nos dio la opción de implementar las acciones que utilizamos anteriormente en Máximo 2.0 y así programar que por ejemplo al presionar $\mathrm{W}$ el personaje camine hacia delante (LAM Mesa, NB Lombana, 2013).

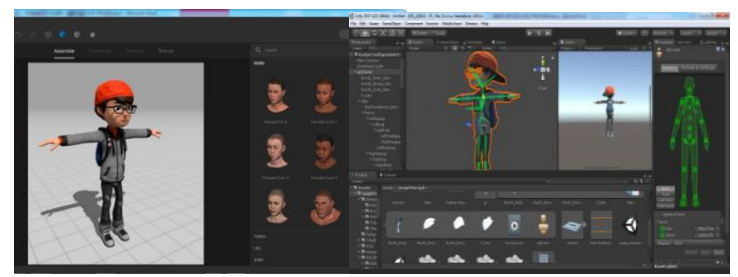

Fig.10. Rigging de personaje mecanim y pruebas en unity $3 d$
Creación de indicadores de las diferentes zonas académico administrativas.

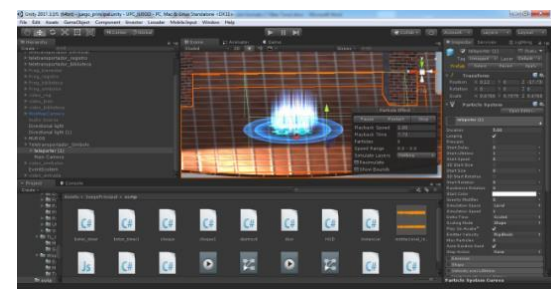

Fig.11. Prueba de funcionamiento indicador de zona en unity $3 d$

Era importante que al usar el OVA éste fuera lo más claro posible al usuario, para esto se realizó la creación de un tipo de indicador el cual tuvo la función de ubicar cada una de las diferentes dependencias académico administrativa, se diseñó un efecto el cual pudiera percibir el usuario a simple vista. El sistema de partículas interno de Unity3d nos permitió simular el indicador con el cual el usuario colisionaría para así activar el video educativo que le daría a conocer las funciones que dicha zona brindaba a la comunidad estudiantil, para lograr esto se desarrolló un script en C\# donde al momento de colisionar con el indicador me activara el video educativo.

\section{Desarrollo de mini mapa de ubicación de las dependencias.}

En esta fase se llevó a cabo el diseño de un mini mapa que permitiría al usuario ubicar fácilmente las distintas dependencias de la universidad, para ello se utilizó una cámara con vista superior, aplicando una textura que reflejara todo el entorno 3d, logrando visualizar toda la infraestructura universitaria. Mediante un Script desarrollado en C\# se logró que la cámara siguiera el recorrido del personaje y reflejara los indicadores anteriormente diseñados.

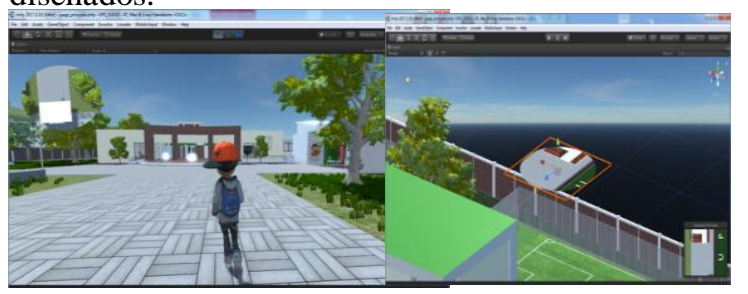

Fig. 12. Prueba de funcionamiento mini mapa en unity $3 d$ 


\section{CAPITULO IV. IMPLEMENTACIÓN DE PRUEBA PILOTO PARA ESTABLECER LA DINÁMICA DEL SOFTWARE CON ESTUDIANTES DE LA UNIVERSIDAD POPULAR DEL CESAR SECCIONAL AGUACHICA.}

Se realizó una Encuesta De Satisfacción a los estudiantes de primer semestre de la universidad popular del cesar seccional Aguachica, pertenecientes a los programas de ingeniería de sistemas, ambiental y agroindustrial. Se seleccionó una población de 144 estudiantes de 230, los cuales presentaron la prueba piloto.

De una población de 230 estudiantes de primer semestre, se realiza el cálculo del tamaño de la muestra.

(1)

$$
n^{\circ}=\frac{Z^{2} x P x}{e^{2}} \quad N=\frac{n^{\circ}}{1+\frac{\circ-1}{N}}
$$

Datos.

$$
\begin{array}{rl}
N=230 \quad 1-\partial=95 \% & e=3 \%=e \\
= & 0,03 \quad P=0,5
\end{array}
$$

(2)

$$
\begin{aligned}
& P+=1 \quad=1-P \quad=1-0,5=0,5 \\
& (1,96)^{2}(\vartheta, 650,5 \quad 0,5) \\
& n^{\circ}=\underline{384} \quad \boldsymbol{n}^{\circ}=\mathbf{8 , 1 6}
\end{aligned}
$$$$
N={ }_{1+\frac{384-1}{230}^{N}=144 \text { oblacion a encuestar }}
$$

\section{CONOCIMIENTOS SOBRE EL OBJETO} VIRTUAL DE APRENDIZAJE.

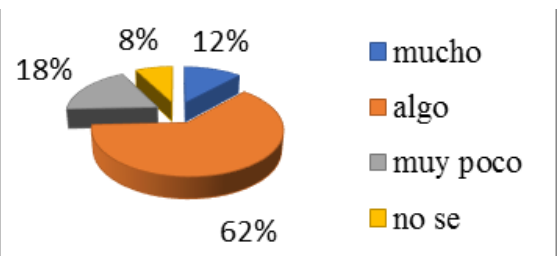

El grafico 1. Se muestra la poblacional encuestada de 144 estudiantes que realizaron la prueba piloto, el $62 \%$ de la población tiene conocimiento de lo que es un objeto virtual de aprendizaje equivalente a 90 estudiantes de la muestra poblacional. Y $8 \%$ de la población no conoce el concepto de ova.

\section{ES NECESARIO QUE LA INSTITUCIÓN CUENTE CON DIDÁCTICAS. HERRAMIENTAS}

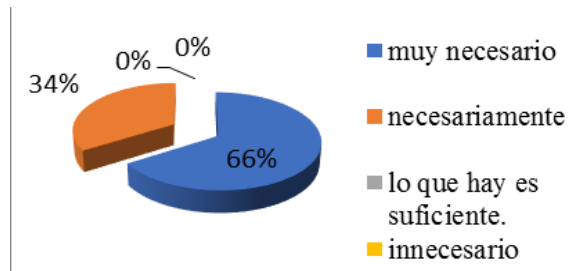

El grafico 2. El $66 \%$ de la población equivalente a 95 estudiantes y el $34 \%$ equivalente a 49 estudiantes de la población encuestada están de acuerdo con que es necesario que la universidad popular del cesar tenga más herramientas didácticas.

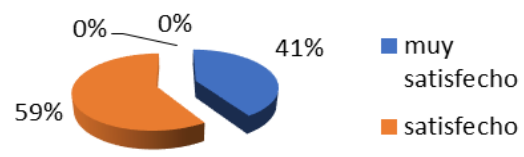

Del grafico 3. El 59\% y $41 \%$ de la población equivalente a 144 estudiantes de la muestra poblacional cree que la ova cumple con las necesidades de la universidad y se encuentran muy satisfecho con respecto al proceso de inducción.

\section{SEGÚN SU PERSPECTIVA, AL USAR EL OBJETO VIRTUAL DE APRENDIZAJE} ESTE ES:

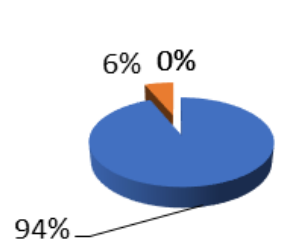

$$
\begin{aligned}
& \text { - muy innovador } \\
& \text { - poco innovador } \\
& \text { " nada innovador } \\
& \text { - no deberia }
\end{aligned}
$$$$
\text { mostrarse }
$$

Del grafico 4. El $94 \%$ de la población equivalente a 135 estudiantes de la muestra poblacional cree que el proyecto ova, presentado es innovador, el $6 \%$ de la población equivalente a 9 estudiantes de la muestra poblacional encuestada piensan que es poco innovador. 
TABLA 3. ESTADÍSTICA DESCRIPTIVA. PERSPECTIVA, AL USAR EL OBJETO VIRTUAL DE APRENDIZAJE

Tabla 3. Estadística descriptiva. perspectiva, al usar el objeto virtual de aprendizaje

\begin{tabular}{lr}
\hline Media & 36 \\
Error típico & 33,0681115 \\
Mediana & 4,5 \\
Desviación estándar & 66,1362231 \\
Varianza de la muestra & 4374 \\
Curtosis & 3,91426612 \\
Coeficiente de asimetría & 1,97572012 \\
Rango & 135 \\
Máximo & 135 \\
Cuenta & 4 \\
Mayor (1) & 135 \\
Nivel de confianza & \\
$(95,0 \%)$ & 105,237489 \\
\hline
\end{tabular}

Tabla 3. Datos tabulados en software Excel. En la siguiente tabulación se muestra una media 36 de la población encuestada, una mediana de 4,5. Con un coeficiente de asimetría del 1,98, con un nivel de confianza del 95\%. Presenta gran satisfacción por la población a la que se le realizo la prueba piloto.

\section{CONCLUSIONES}

$>$ La implementación de este objeto virtual de aprendizaje genero ayuda en los estudiantes y contribuyo a innovar en el proceso de enseñanza, esta herramienta les brindo a los profesores una ventaja significativa en cuanto a mantener a los partícipes activos de las actividades y poder fortalecer los conocimientos adquiridos durante la clase. Este videojuego al ser utilizado para una función educativa provoco en los estudiantes dos efectos los cuales son divertirlos y a la vez el de enseñarles, de tal forma que el aprendizaje que se genere sea significativo, por lo cual, no será olvidado por el estudiante y perdurará a través del tiempo.

> Para dar respuesta a los objetivos planteados concluimos que la estimación de la muestra poblacional encuestada con respecto a los recursos didácticos del Objeto Virtual de Aprendizaje, se deben mejorar. La población del $35 \%$ y $10 \%$ coincidió con que los videos, escritos y gráficos, deberían tener una mejora. En cuanto a la estimación en correlación con el otro $45 \%$ de la población encuestada no manifestó ningún cambio necesario para la ova. Se realizó un mejoramiento, teniendo en cuenta el mayor índice de población del 55\% que realizo la encuesta. Corrigiendo así los recursos disponibles como videos de presentación donde se da a conocer la información de cada dependencia, revisando los textos escritos y mejorándolos. Con respeto a los errores de gráficos fueron solucionados, los cuales correspondían a errores de colisiones, donde el personaje principal chocaba con los diferentes objetos ocasionando un bucle de caída.

\section{REFERENCIAS}

Aguas del cesar, municipio de Aguachica cesar [base de datos en línea]:http://aguasdelcesar.com.co/index.php? option $=$ com_content $\&$ view $=$ article $\& i d=81: \mathrm{m}$ unicipio-deaguachica\&catid $=3$ : municipios $\&$ itemid $=51$ fecha: 19 de marzo de 2017 hora 13:42 pm.

Adobe fuse cc (beta), fuente en línea [https://helpx.adobe.com/beta/fuse/faq.html]: fecha 5 febreo 2018, hora 4:53 pm.

Blender guía 2.3, la suit abierta de creación 3d, modificada por leono. [base de datos en línea]:

http://www.futureworkss.com/tecnologicos/in formatica/tutoriales/manual_de_blender.pdf, pag:2,5,6; fecha 5 de septiembre de 2017 hora 11:42 pm.

Constitución política de Colombia 1991, capitulo 2 , de los derechos sociales, económicos y culturales. [base de datos en línea]: http://www.alcaldiabogota.gov.co/sisjur/norm as/norma1.jsp?i=4125 fecha: abril 9 de 2017 hora 1:30 pm.

Desarrollo de un videojuego educativo basado en Unity 3d y Blender 3d, para estimular el autoaprendizaje y apoyar el proceso de enseñanza de tecnología en educación secundaria

http://dspace.ucundinamarca.edu.co:8080/xml ui/bitstream/handle/123456789/736/tecnogam e.pdf? sequence $=2 \&$ isallowed $=y$, fecha 23 enero 2018, hora 2:30 pm.

J. Guzmán-Luna, I. D. Torres, J. F. Alvarez. (2014). Propuesta de un generador de aplicaciones educativas basadas en televisión digital usando arquitectura de cómputo en la nube. REVISTA 
COLOMBIANA DE TECNOLOGÍAS DE AVANZADA, ISSN: 1692-7257. 1(23).

J. Guzmán Luna, I. Torres and J. Alvarez. (2014). Propuesta de un generador de aplicaciones educativas basadas en televisión digital usando arquitectura de cómputo en la nube. REVISTA COLOMBIANA DE TECNOLOGÍAS DE AVANZADA, vol. 1, no. 23, 2014.

JEG Plaza, MAR Nuñez, (2017), Formación en competencias específicas para la industria del software colombiano. Experiencias del uso del aprendizaje basado en proyectos Revista Colombiana de Tecnologías de Avanzada ISSN: 1692-7257.

L. A. M. Mesa, N. B. Lombana. (2013). La robótica educativa como instrumento didáctico alternativo en educación básica. REVISTA COLOMBIANA DE TECNOLOGÍAS DE AVANZADA, ISSN: 16927257. 2(22).

L. Mesa Mesa and N. Barrera Lombana. (2013). LA ROBÓTICA EDUCATIVA COMO INSTRUMENTO DIDÁCTICO ALTERNATIVO EN EDUCACIÓN BÁSICA. REVISTA COLOMBIANA DE TECNOLOGÍAS DE AVANZADA, vol. 2, no. 22.

L Márquez, YA Lara, F Ángulo, (2017), Prototipo de control de acceso a aulas y registro automático de asistencia Revista Colombiana de Tecnologías de Avanzada ISSN: 1692-7257.

L.O. T. Tellez, M. P. S. Delgado, W. M. R.Contreras. (2014). Modelo de interventoría de tecnologías de información en el área de conocimiento de la gestión del alcance de pmbok ${ }^{\circledR}$ y alineado con iso 21500 y cobit ${ }^{\circledR}$. REVISTA COLOMBIANA DE TECNOLOGÍAS DE AVANZADA, ISSN: 1692-7257. 1(23).

Tangarife Tellez, L., Sánchez Delgado, M., \& Rojas Contreras, W. (2014). Modelo de interventoría de tecnologías de información en el área de conocimiento de la gestión del alcance de pmbok® y alineado con iso 21500 y cobit ${ }^{\circledR}$. REVISTA COLOMBIANA DE TECNOLOGÍAS DE AVANZADA, 1(23).

Manual de creación de videojuego con unity 3d, iman ouazzani; juan peralta donate. [base de datos en línea]: $\quad$ https://e-

archivo.uc3m.es/bitstream/handle/10016/1634

5/pfc_iman_ouazzani.pdf?sequence $=1$, pag.

$21,22,23,24$. Fecha 5 de septiembre de 2017 hora 2:00 pm.
M. C. Bonfante, A. Castillo. (2014). Integración de sistema multi-agente, ontologías y procesos de negocios como marco tecnológico de la estrategia "gobierno en línea". REVISTA COLOMBIANA DE TECNOLOGÍAS DE AVANZADA, ISSN: 1692-7257. 1(23).

Bonfante, M., \& Castillo, A. (2014). Integración de sistema multi-agente, ontologías y procesos de negocios como marco tecnológico de la estrategia "gobierno en línea". REVISTA COLOMBIANA DE TECNOLOGÍAS DE AVANZADA, 1(23).

MR Contreras, MS Delgado, (2013), Arquitectura de software para el servicio de soporte de tecnología de información basada en servicios web. Revista Colombiana de Tecnologías de Avanzada ISSN: 1692-7257.

Simuladores de cirugías colaborativo, laboratorio de investigación en realidad virtual. Universidad eafit, realidad virtual apoyando el entrenamiento remoto y en equipo de procedimientos quirúrgicos. [base de datos en línea]:

http://arcadia.eafit.edu.co/html/simuladorcola borativo.html. Fecha: 20 de marzo de 2017 hora 10:42 am.

Tendencias de la ingeniería, revista electrónica de ciencia, tecnología, sociedad y cultura. Issn 2174-6850. Divulgando conocimiento desde 1988. [base de datos en línea]:http://www.tendencias21.net/crean-unsimulador-en-3d-para-el-aprendizaje-virtual- delmedioambiente_a3565.html,51 fecha: 19 de marzo de 2017 hora 13:42 pm.

Unity 3d, iman ouazzani; juan peralta donate. [base de datos en línea]: https://earchivo.uc3m.es/bitstream/handle/10016/1634 5/pfc_iman_ouazzani.pdf?sequence $=1$, fecha 5 de septiembre de 2017 hora 2:00 pm.

Imagen tomada por los autores. Software de creación de video juegos, licencia para estudiantes, link del software.

Imagen tomada por los autores. Aplicación web de animación 2d, licencia Startup activa, link de la aplicación: https://www.animaker.es, Hora 9:30 pm, Fecha 30 marzo 2018.

Imagen tomada por los autores. Software libre, licencia de código abierto, link del software: https://www.blender.org/. Hora 8:30 pm, Fecha 30 marzo 2018. 